\title{
Testicular cancer presenting as a red swollen lid
}

\author{
J A HAM' AND N J CARR ${ }^{2}$
}

From the ${ }^{1}$ Department of Ophthalmology, Royal Air Force Hospital, Wegberg, West Germany, and ${ }^{2}$ Department of Histopathology, Institute of Pathology and Tropical Medicine, Royal Air Force Halton, Aylesbury, Bucks HP22 5PG

SUMMARY A case is reported in which a testicular cancer presented as a metastatic deposit in the eyelid. The histological appearances of both the metastasis and the subsequently detected primary were diagnostic of malignant teratoma trophoblastic (MTT). After comments on the testicular tumour, metastases occurring in the eye and adnexa are discussed.

A 19-year-old Army sapper was referred to the Ophthalmic Department at the Air Force Hospital in Germany from a nearby station medical centre with a five-day history of a painless red and swollen left upper lid. He had no other compaints, and there was no relevant past ocular or medical history. On examination the lateral aspect of the left upper lid showed a very vascular swelling. On lid eversion the subtarsal tissue was soft, friable, and bled to touch. There was no bulbar conjunctival injection, and the globe assessment, which included a fundal examination after mydriasis, was normal. Preauricular lymph nodes were not enlarged. The affected lid was infiltrated with $1 \%$ lignocaine, and piecemeal biopsies were taken from the subtarsal tissue and from just anterior to the lash line. Histological examination revealed metastatic tumour (see below).

Correspondence to Flight Lieutenant N J Carr.

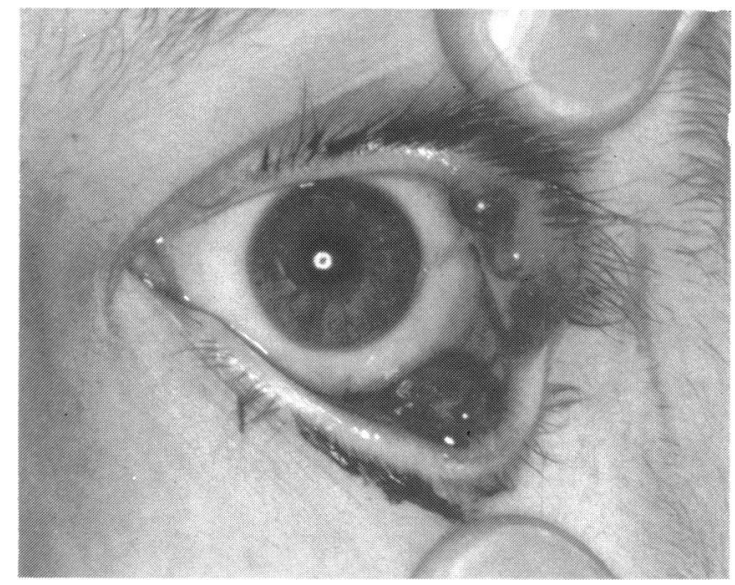

Fig. 1 Left eye: the eyelid lesions on the day of admission.
Four days later the patient was found to have spontaneous bleeding from his left eyelids. An increase in the size of the upper lid swelling had occurred, and a fresh haemorrhagic lesion in the lower fornix was noted (Fig. 1). A verbal report identifying the lid lesion as metastatic MTT was available, and the patient was admitted for further investigations immediately. He denied any lassitude, anorexia, or weight loss. He also denied any testicular abnormality, even on direct questioning. On examination he was a hirsute young man with no gynaecomastia or palpable lymphadenopathy. The

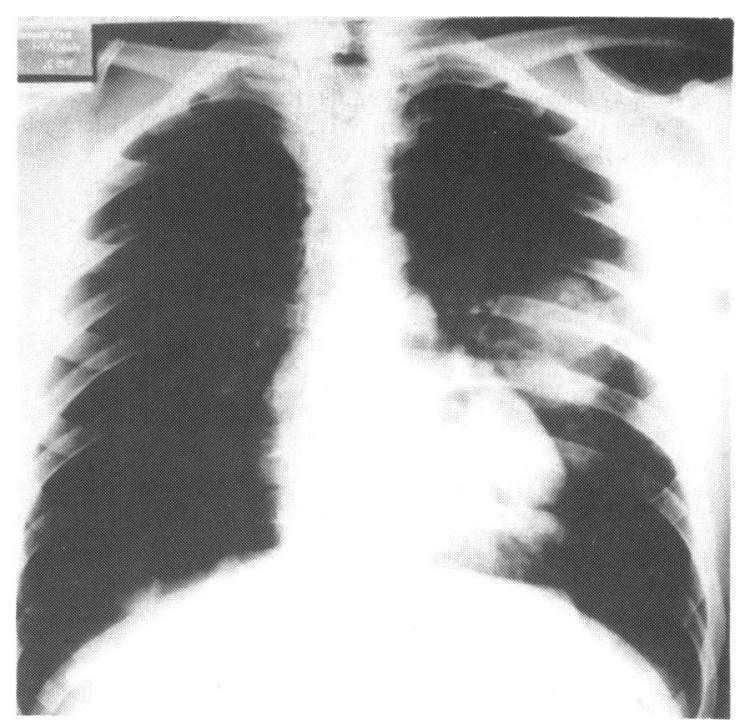

Fig. 2 Chest radiograph showing round opacities in the left mid zone and right lower zone. 


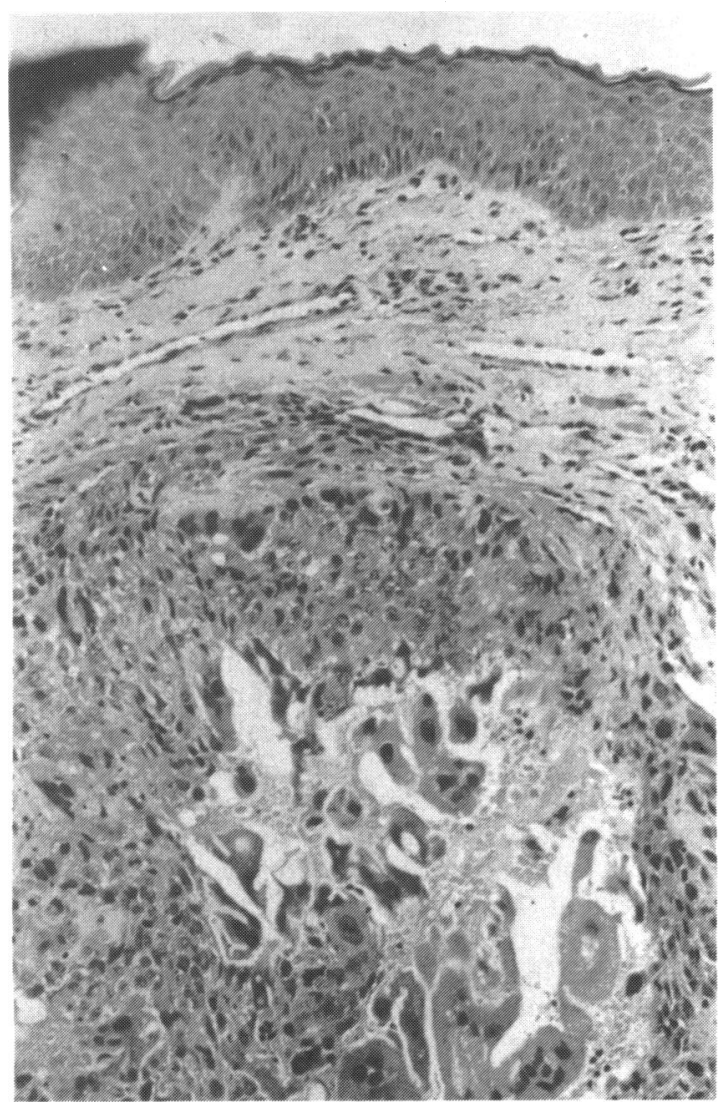

Fig. 3 Section from an eyelid lesion showing metastatic MTT within the dermis. Multinucleate syncytial cells overlying cytotrophoblastic cells are clearly visible in relation to a blood filled space. The epidermis is seen in the upper part of the figure. $H$ and $E, \times 110$.

only abnormality on clinical examination was an obviously enlarged, nodular, non-tender, left testicle. Laboratory investigation revealed a haemoglobin concentration of $7 \cdot 8 \mathrm{~g} / \mathrm{dl}$, and his chest radiography showed multiple round opacities in both lung fields (Fig. 2). An urgent orchidectomy was performed in Germany and histological examination confirmed the testis as the primary site of the MTT. Four days postoperatively he returned to the United Kingdom for further treatment in an oncological unit.

\section{HISTOPATHOLOGICAL FINDINGS}

The metastatic tumour in the eyelid was located in the dermis and composed of trophoblastic tissue surrounding blood spaces (Fig. 3). Immunohistochemical stains ${ }^{1}$ showed the malignant cells to be strongly immunoreactive with antibody directed against human chorionic gonadotrophin (Fig. 4) and

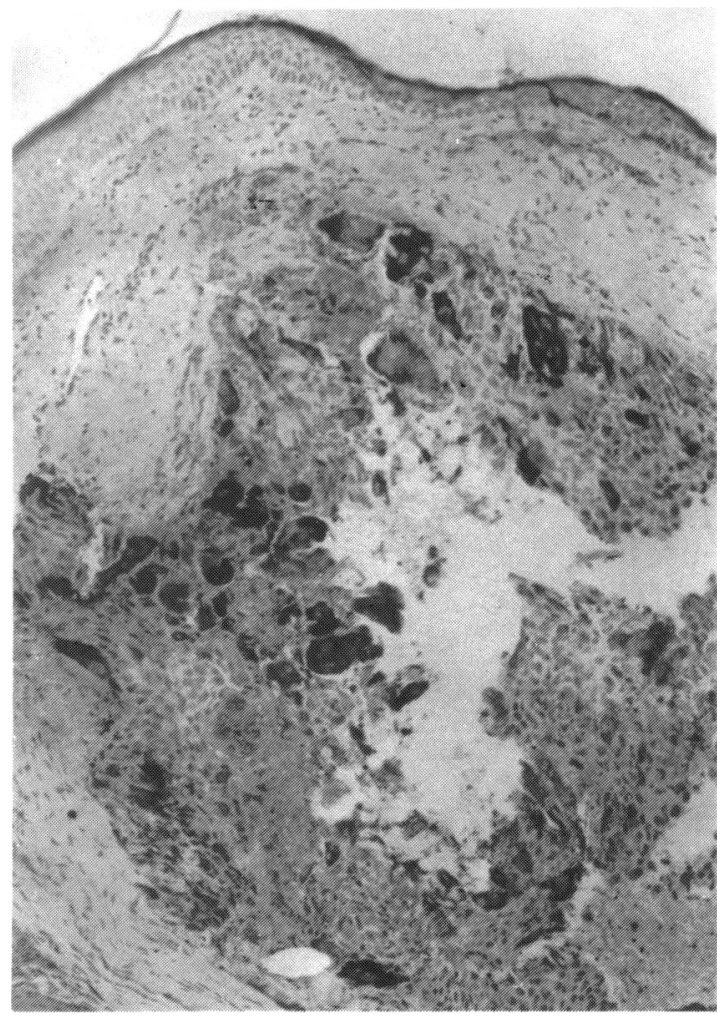

Fig. 4 Eyelid lesion: the syncytiotrophoblasts show strong immunoreactivity with antibody directed against $H C G$. Immunoperoxidase, $\times 67$.

cytokeratin, but negative for $\alpha$-fetoprotein and S100 protein.

The testicular lesion was composed of extensive areas of necrosis and haemorrhage within which tumour tissue was sparsely distributed. Syncytiotrophoblastic and cytotrophoblastic elements were clearly visible in relation to vascular spaces (Fig. 5). Also present were areas of organoid differentiation, which included cysts lined by squamous epithelium and bland cartilage, and partially differentiated areas. No yolk sac components were identified.

\section{Discussion}

Testicular tumours are almost invariably primary and malignant, presenting most commonly between the ages of 20 and $40 .{ }^{2}$ Approximately $65 \%$ of patients present with a painless swelling, $20 \%$ complain of pain with or without swelling, and $10 \%$ of tumours are asymptomatic and detected on routine clinical examination. In less than $5 \%$ of cases does the patient present because of metastases. Initially metastases tend to occur in the para-aortic nodes and lungs; pulmonary spread may be detected by routine 


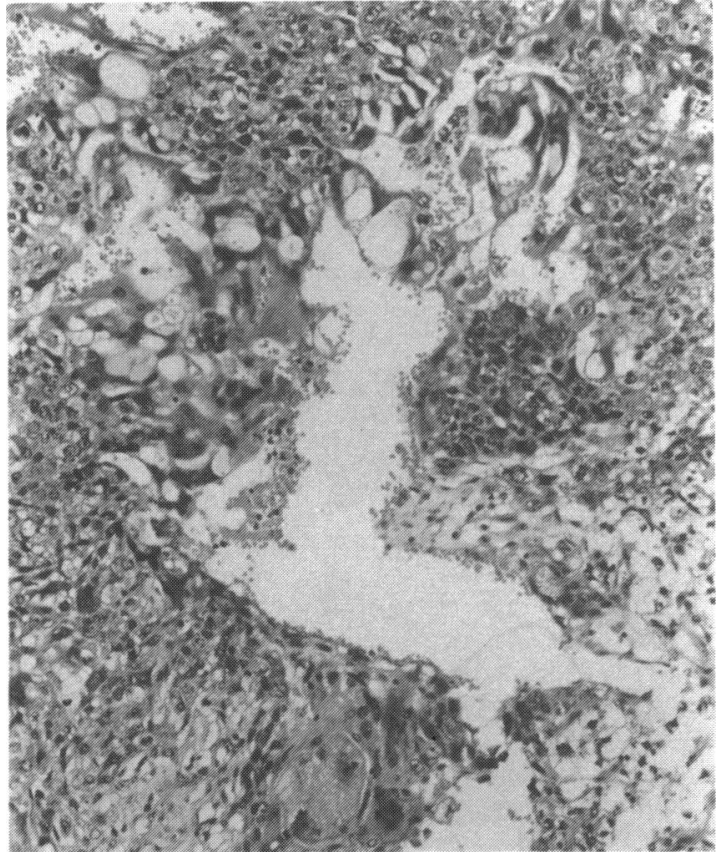

Fig. 5 Part of the testicular tumour showing similar morphology to that seen in the eyelid lesion. $H$ and $E, \times 93$.

chest $x$-rays. A dermal metastasis is an unusual presentation for a testicular tumour. In this case the primary could have been detected earlier, but the patient failed to present in spite of marked painless swelling of one testicle.

Teratomas account for approximately $30 \%$ of testicular tumours, and about $4 \%$ of these are MTT, also known as testicular choriocarcinoma. The histology of teratomas varies from well differentiated lesions, commoner in childhood, to the more malignant lesions graded intermediate or undifferentiated. According to the British Testicular Tumour Panel the following criteria are required for the histological diagnosis of MTT: ${ }^{3}$ the presence of recognisable syncytiotrophoblastic and cytotrophoblastic elements, and their arrangement in a papillary pattern. Both the eyelid lesion and the testicular tumour satisfy these criteria. The appearances closely resemble those seen in gestational choriocarcinoma in the female.

Most metastases encountered by the ophthalmologist are intraocular or orbital; metastatic deposits in the eyelid are rare. Only 15 cases of secondary tumour in the eyelid were seen at the Mayo Clinic between 1922 and $1969 .{ }^{4}$ The lesions in this series tended to occur as a manifestation of generalised carcinomatosis, and in only two cases (both of which were adenocarcinoma of the stomach) were the eyelid lesions the presenting complaint. Deposits in the eyelid from primaries in the breast, skin (malignant melanoma), and lung have been reported in particular, but other primary sites include colon, stomach, prostate, thyroid, kidney, and cervix..$^{45}$

Metastatic lesions of the eyelid are said to take one of three forms. ${ }^{6}$ Firstly, solid subcutaneous nodules occur which may be mistaken for chalazia. Secondly, an ulcerating lid lesion may occur which may mimic a basal cell carcinoma clinically. Thirdly, a diffuse swelling may result with non-tender thickening and induration of the eyelid. This case was unusual in that the trophoblastic nature of the deposit caused bleeding on eversion of the lid. With metastatic tumours of the eyelid the vascular reaction may suggest an inflammatory lesion rather than a neoplasm, and in this case the initial referring medical officer had made a provisional diagnosis of an inflammatory lesion.

In contrast to eyelid metastases, metastases to the eye and orbit are well described in the literature. The majority are blood borne following pulmonary involvement. In a review of 220 cases of carcinoma metastatic to the eye or orbit, ${ }^{7}$ which comprised all cases on file in the Registry of Ophthalmic Pathology at the Armed Forces Institute of Pathology, Washington, DC, 28 patients had orbital metastases and in 17 cases symptoms of the orbital lesions preceded detection of a primary tumour elsewhere in the body. One patient in this series was a 20 -year-old male with intraorbital metastasis from a testicular choriocarcinoma.

We thank the Director General of Medical Services, Royal Air Force, for permission to publish this paper, and Mrs Connie Simmons for preparing the manuscript.

\section{References}

1 Risdon RA. Germ cell tumours of the testis. J Pathol 1983; 141: 355-61.

2 Mostofi FK. Testicular tumours, epidemiologic, etiologic and pathologic features. Cancer 1973; 32: 1186-201.

3 Pugh RCB, Cameron KM. Teratoma. In: Pugh RCB, ed. Pathology of the testis. Oxford: Blackwell, 1976: 199-244.

4 Riley FC. Metastatic tumours of the eyelids. Am J Ophthalmol 1970; 69: 259-64.

5 Bloch RS, Gartner S. The incidence of ocular metastatic carcinoma. Arch Ophthalmol 1971; 85: 673-5.

6 Duke-Elder S, MacFaul PA. The ocular adnexa. In: Duke-Elder S, ed. System of ophthalmology. London: Kimpton, 1974: 536-8.

7 Ferry AP, Font RL. Carcinoma metastatic to the orbit. Mod Probl Ophthalmol 1975; 14: 377-81; 1970; 69: 259-64.

Accepted for publication 3 September 1987. 